\title{
Carnets
}

Revue électronique d'études françaises de l'APEF

Première Série - 3| 2011

$L^{\prime}($ In)vraisemblable

\section{Dégivrage Technique}

\section{Marc-Ange Graff}

\section{(2) OpenEdition}

\section{Journals}

Édition électronique

URL : http://journals.openedition.org/carnets/6218

DOI : 10.4000/carnets.6218

ISSN : 1646-7698

Éditeur

APEF

Édition imprimée

Date de publication : 2 janvier 2011

Pagination : 191-207

\section{Référence électronique}

Marc-Ange Graff, « Dégivrage Technique », Carnets [En ligne], Première Série - 3 | 2011, mis en ligne le 18 juin 2018, consulté le 24 septembre 2020. URL : http://journals.openedition.org/carnets/6218 ; DOI : https://doi.org/10.4000/carnets.6218

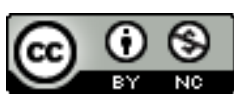

Carnets est mis à disposition selon les termes de la licence Creative Commons - Atribution - Pas d'utilisation commerciale 4.0 International. 


\section{DÉGIVRAGE TECHNIQUE}

MARC-ANGE GRAFF

Université des Açores

mgraff@uac.pt 
Ainsi vivions-nous: à froid. Luiza Neto Jorge 
Longtemps, je me suis rêvé frigidaire. C'est fini maintenant: ma vocation s'est liquéfiée, et je vis aujourd'hui, comme les hommes, dans un lyrisme tiède aux ascenseurs climatisés, sous une pluie d'avril qui ne mouille que les fous. Je reçois du courrier, je souris de la plume et des lèvres: vertu fertile et molle des gens normaux et des coureurs cyclistes. Mes blocs de froid et de silence se sont ouverts sur une chair vivante, musique d'aérogare, antennes quelque peu anémiées.

Je ne prétends dire ni comment ni pourquoi j'ai changé. Je ne souhaite rien d'autre que de noter, de serrer un peu plus les nœuds d'une métamorphose d'abord presque insensible, à partir de quelques menus faits, ni glissades ni précipices, dont il m'a bien fallu convenir qu'il s'agissait de signes, d'étapes, alors que je les ai ressentis tout d'abord comme des contrariétés, comme des retours fugaces de mon passé, d'un temps que je n'avais jamais désiré retrouver, mais que je contemplais de loin avec la solidité aride de la mort, comme dans les jardins publics les bancs de pierre sont les témoins muets des joutes enfantines. 
Sur la plage déserte, sous ce triste ciel d'automne marneux, le champagne du sable avait déjà son goût d'hiver, ce goût de vieux cidre éventé que je connais si bien. L'avaler ne coûte guère pourtant, le recracher est inutile: sa fadeur légèrement amère demeure dans la bouche jusqu'au printemps suivant, jusqu'à la mort de la vie morte.

Avant cet office distrait, j'avais parlé longuement à une amie spectrale du carnier plat de ce chasseur toujours bredouille qui suivait à la trace, aux traces de la trace, le gibier de la saison passée. Pour elle, j'évoquais la splendeur des garennes, à la fin de l'été, quand je me faisais lièvre, chevreuil ou marcassin. Pour elle, je déplorais les cartouches perdues, à l'aube froide, sur la gelée blanche, quand j'éclatais en crépitements sourds.

Comme tous les ans à cette époque, la plage était semée de pédalos rouillés qui étaient des steamers, de crocodiles désaffectés, de longues lanières d'algues, de traces de jeux d'enfants et de bidons d'essence qui faisaient les cent pas. J'aimais cette mélancolie sans bornes de la mer, ses vagues, comme les fantômes régurgités de jeunes filles en fleurs.

J'étais très loin de moi, plus loin que tous les horizons, plus loin que toutes les eaux-vives que l'été ramenait chaque année sur les côtes, et que chaque année l'équinoxe d'automne renvoyait aux traités de zoologie fantastique. J'étais loin comme une dent de lait, et ce n'était guère que par pusillanimité puérile, par de fugaces accès de terreur de la paralysie totale que je songeais parfois à mon retour à moi et aux gestes du monde.

(Le sable des plages, quoi qu'on en pense, possède lui aussi son architecture. Quelques infimes crustacés me l'avaient enseignée en silence. Je revenais souvent à cette idée, que j'aimais d'autant plus qu'il m'était difficile d'en tirer des conséquences pratiques, difficile même de me l'imaginer. Il s'agissait pour moi d'un savoir sans emploi ou, mieux 
encore, d'une image poétique qui ne trouverait jamais son poème: une figure du loin, de l'inutilité sans joie. Et contradictoirement, le sable avait aussi un goût, des goûts divers selon la saison, les calculs de résistance des matériaux n'avaient rien à y faire, et les lois de la perspective se défont entre les interstices des molaires. Ce jour-là, le sable avait un goût d'hiver et son architecture impalpable occupait mon esprit: j'étais seul, fort loin de tout commerce et usage, loin de la place Blanche où je m'étais connu.)

J'avais fui. J'ignore encore quoi et pourquoi, et le mot fuite est sans doute trop fort. Certes, je n'aimais plus les anciens parapets; mais les dérades, les désirades, les poteaux de couleurs ne figuraient pas non plus sur ma fiche technique. Pourquoi avais-je choisi, d'ailleurs, cette île de brouillard plutôt qu'une autre? II ne m'en reste aucune idée.

(Treize ans d'exil, et me voici sur cette plage que je goûte pour la centième fois, comme pour mieux savourer le triste goût de mon absence, comme pour insister sur mon âcre vertu d'anachorète vieillissant. Et il faut bien que ce récit languisse, qu'il épouse en son rythme trop lent le lent déroulement de mon absence à moi, car ces treize ans en sont peutêtre quinze, ou bien dix-huit ou vingt, je ne sais même plus, et j'ignore si ma vie s'est poursuivie, déroulée dans cette île, ou bien si une machine à répéter les jours n'avait pas pris possession de la mer et du ciel, des bêtes et des arbres, et des quelques fantômes de chair fraîche que je croisais et recroisais au détour du chemin. II est si facile d'être son propre singe...)

En cet automne, pourtant, bien malgré moi il m'advint quelque chose. Ce ne fut tout d'abord qu'une vague impression, un fourmillement pressé des grains de sable noir qui coulaient de mes mains, comme si leur chute s'accélérait imperceptiblement sans que mes doigts se fussent ouverts. Ma communion aride avait déjà pris fin, peu m'importait d'ailleurs, et je ne cherchai pas à retenir leur chute. Les mains vides soudain, il ne me restait plus qu'à entamer un autre soliloque.

II s'agissait d'un de mes mythes récents, que je n'avais pas encore établi fermement dans ma tradition orale, où les ronds de sorcières des champignons, comme dans un jeu de piste infernal et très doux, entraînent le cueilleur tout au fond de lui-même en un labyrinthe qui va s'étrécissant toujours, jusqu'à le réduire à l'immobilité complète, et dont il ne peut sortir qu'en se desséchant sur place, tous ses désirs lui échappant alors et 
s'éparpillant au vent comme des spores. Le ton désespéré manquait encore, et je comptais sur ce nouvel essai pour m'en approcher quelque peu, tout en sachant très bien que cette fois non plus je ne parviendrais pas à émouvoir véritablement mon public, composé de moimême et de moi.

Mais c'est tout d'abord la ferveur qui fut au rendez-vous, et puis la joie et l'allégresse, quand les désirs s'envolèrent en dansant, laissant la pauvre statue de poussière pétrifiée toute à son inertie recluse.

Le soir tombait, il était temps que je regagne ma cuisine, son carrelage de faïence uniforme. 
Tout émaillée de blanc, ma porte s'ouvrait peu. Très sûr de ma lenteur, impassible comme un portrait de pape, impeccablement propre, toujours parfaitement rasé, je fabriquais du froid. En moi, et tout autour de moi. Un navire cargo m'avait déchargé sur le port. Mes jours étaient carrés et mes nuits étaient ternes: c'était bien.

L'automne ici dure six mois, quelquefois huit: certains trouvent le temps de lire tous les livres, et de s'amuser des tristesses de la chair. La pluie est tiède, comme un crapaud cueilli au crépuscule, mais elle dure toute la journée. L'engourdissement succède assez vite au sommeil, que l'assoupissement précède de peu. II est, bien entendu et comme partout ailleurs, quelques brutes qui s'agitent, parlent trop haut, font des projets; comme partout ailleurs, on les isole au Parlement ou on les parque à l'Université: ils y nuisent à tous sans déranger personne. En somme, sans l'avoir vraiment élue, j'avais bien choisi mon île: je n'y étais qu'un peu plus morne que les autres, qu'un petit peu plus préoccupé de rien.

Ancré, solidement ancré sur cette portion de terre dérisoire, solidement ancré dans ma ténacité morose. N'eût été le fait que j'étais né ailleurs, que je parlais (quand je parlais) une autre langue, personne n'aurait remarqué ma présence. J'ai d'ailleurs rapidement cessé d'être insolite, comme les nouveautés déjà flétries d'anciens salons des arts ménagers. Je fabriquais du froid: pas ce froid intense et presque asymptotique que savent produire les laboratoires modernes, mais un froid à ma mesure, un froid modeste et à usage individuel, tout juste bon à solidifier les cubes de glace apéritifs, un petit froid ne permettant qu'une mise en conserve raisonnée.

Manque d'ambition peut-être, ou tempérament peu enclin au lyrisme: je n'ai jamais souhaité être un congélateur. 
Dois-je dire, ou sais-je même comment j'occupais mes journées? En fait, j'ai plutôt l'impression que les jours m'occupaient: le temps prenait possession de moi, m'envahissait comme une terre conquise. On me dira sans doute que c'est humain, que nous sommes tous les jouets et les victimes du temps, qu'il nous entraîne et nous ravage. Mais ce n'est pas de cela qu'il s'agit: le temps passe sur les hommes, il n'est sur eux que passage, vestiges, alluvions et moraines. Moi, je l'emmagasinais: il entrait en moi sans même ouvrir la porte, je l'accueillais et l'installais, et il ne sortait plus.

Était-ce un temps abstrait? ou un temps vide? Vidé plutôt. Le temps en effet convoie des événements, et son passage en nous enrichit et dépossède tout ensemble: les événements passés sont du côté des pertes, l'expérience du côté des profits.

Je n'ai jamais été homme de profits. Je n'aimais guère les événements. 
J'avais pourtant des rites, quelques gestes, des mots. J'inventais des figures de la mort, j'observais le lointain s'effacer dans la brume, je recensais des traces. J'aimais aussi peupler mon univers et pour ce faire, je m'entourais de feuilles de papier à cigarettes, de buvards, de taille-crayons et d'encriers. De vieilles photographies dansaient la gigue dans un tiroir que je n'ouvrais jamais. Parfois, je lavais aussi la vaisselle.

Mes mots comptaient, je racontais. Je me racontais des histoires dont les plus réussies se transformaient en mythes, bien que je n'en fusse pas toujours le personnage central. Le narrateur mourait souvent au milieu de l'histoire, qui ne s'en continuait pas moins sur un ton plus feutré, et comme il aimait ça, il racontait souvent: mourir, c'est raconter un peu. J'étais multiple et un, comme un lot de T-shirts vendus à la criée.

On prétend parfois que les amitiés de voyage prennent fin dès l'arrivée au port. Pour moi, je m'étais pris d'affection pour un gros clou rouillé que j'avais ramassé sur le pont du cargo. Il partagea ma solitude: je le baignais tous les matins, avant de le laisser s'ébrouer sur le rebord de la fenêtre, où un soleil toujours timide parvenait parfois à le sécher. Je lui avais offert un pied à coulisse et tous les soirs, en le mesurant, je commentais pour lui les progrès de la rouille.

Ce fut un second signe: un triste jour, la rouille cessa de progresser.

Je porte désormais le vieux clou à mon cou, comme on porte une croix.

D'or. 
La vieillerie érotique avait une bonne part dans mon régime alimentaire. J'appris à fréquenter la plage, à y venir très tôt le matin, dès les premiers beaux jours, si rares qu'il n'en faut perdre aucun. Je tenais un cadastre: à la fin de l'été, je savais avec certitude tous les emplacements où s'étaient allongées des filles, et si c'était de préférence sur le dos ou le ventre. J'aurais pu dessiner une carte, où les rares taches exemptes de sueur féminine auraient figuré des déserts, des no man's lands, des territoires hostiles. L'automne revenu, je choisissais les lieux les plus assidûment peuplés pour y communier stérilement avec la foule de mes désirs géométriques, qu'avaient balayés les marées de syzygie.

Au crépuscule, je faisais ma récolte. C'était le temps béni où les apôtres de l'écologie prêchaient encore dans le désert, et n'étaient nullement relayés par la voirie municipale. Je ramassais les flacons vides de lotion solaire, les rognures d'ongles, les mamelons aux larges aréoles nacrées des berniques, tous les ustensiles de la pêche amoureuse, détritus desséchés, métaphores de cyprine et de frai de grenouilles. Je rentrais avec mon trésor.

Ma cuisine était un musée d'odeurs. Sur des étagères, aussi soigneusement étiquetée que les livres d'une bibliothèque, ma collection répandait des parfums engageants d'ambre solaire et de monoï, dont les lourds effluves m'assoupissaient du sommeil sans espoir de la marchandise hors d'usage. Ils se diffusaient et se diluaient lentement, semaine après semaine, et j'aimais ces relents fades de désirs avortés, d'amours surannées et lointaines par lesquels l'odorat communiait avec l'œil. Plus sûrement que le calendrier, mieux que le thermomètre ou le journal, mes troublantes étagères m'informaient des approches de l'hiver. Je remplissais alors mes flacons, comme on conserve un animal dans le formol ou comme on scelle un armistice, de l'artillerie désamorcée et minuscule de graines de balsamine, pour ne plus jamais y toucher: mon cimetière végétal et marin n'avait pas vocation de fleurir. 
Nous n'irons plus au bois, les lauriers sont coupés. Comme une pluie de sauterelles ou une marée de touristes, les poubelles de plastique ont envahi les plages. L'amour est recyclé, et ses langueurs fondues en une hygiène abstraite: ma solitude forgée au soufflet de l'absence en est devenue apocryphe. 
J'ai voulu renforcer mon étanchéité. Le mur de Berlin était à vendre en demi-gros et en détail. On promettait sous peu le pont de Mostar et la muraille de Chine, j'allais pouvoir me calfeutrer.

Les premiers envois me donnèrent de l'espoir. C'était des pierres relativement neutres, guère bavardes, fort bien conditionnées. Elles m'allaient comme un costume parfaitement coupé, quoiqu'un peu lourd: confection soviétique, me disais-je.

Je devins taciturne et me satisfaisais de quelques mots, style officiel de liturgie laïque mais intimiste. Les bruits du monde ne passaient plus ma porte, le loin redevenait lointain, il faisait bon sous mon amas de pierres.

Lorsque parut pourtant, à un angle du mur, la première saxifrage, l'une après l'autre, je résolus de chauler les pierres à diverses reprises. Tous mes murs furent blancs, enfin blancs comme des suaires sur la neige. Le sol était blanc lui aussi, blanche ma voix et blanche ma douleur: ce ne fut qu'un répit.

Ma douleur était blanche, j'avais une douleur. Je m'en souviens maintenant et je sais que c'est elle qui m'a rendu au monde, à ses rides vénérables, bien que je ne l'aie guère ressentie sur le coup. Quelque chose m'habitait, quelque chose comme un moi agité qui murmure, qui s'essouffle, qui tremble. Quelque chose qui voulait, ne voulait pas sortir. Et la saxifrage en était le symbole: elle insistait, on aurait dit un timbre au coin d'une lettre en retard.

Comme un salpêtre ou une moisissure, le monde avait recommencé de s'infiltrer en moi. 
Faire du froid, quoi qu'on en pense, n'est pas de tout repos. II faut astiquer son carrelage, épousseter ses encoignures, apprendre tous les gestes de l'immobilité. Dormir ne représente qu'un péril relatif, lorsque vient le sommeil avec sa chape de plomb.

C'est le problème du moteur qui est le plus complexe: le bruit doit être permanent et monotone, si monotone et permanent qu'il paraisse silence et force contenue. Les frigidaires ont de larges épaules, mais ne se battent pas. On ne repère leur puissance que dans leurs bâillements.

Je les ai souvent observés dans mon enfance. J'épiais, j'essayais de deviner leurs mouvements rentrés, leurs pensées marmonnées, leurs rêves. J'allais les surprendre la nuit, comptant sur une distraction autorisée par le sommeil de la maison: jamais je n'en ai pris un seul en faute.

Ce qui sans doute me fascinait le plus, c'était que leur hostilité si évidente à la douce tiédeur domestique ne se traduisît jamais par un brusque flot de révolte, par un jet de glaçons et de grêle martelant soudain les murs de la cuisine. Je les imaginais comme de petits volcans inverses, dont la lave gelée si patiemment accumulée devait fatalement un jour se muer en une pluie concrète. J'ignorais encore que leur patience avait pour nom obstination, que leur air sec et rêche était leur seule sauvagerie, lucide et cultivée avec rigueur. Comme des chats en négatif, ils ronronnaient leur désaccord, ils polissaient leurs griffes, mais les gardaient à l'intérieur. Je les avais nommés: les philosophes hermétiques.

Depuis, beaucoup de livres ont coulé sous les ponts, moins pressés par le flot que par la foule de leurs semblables. La quadrature m'est venue lentement, sans choix délibéré. À en juger d'ailleurs par la géographie, j'ai dû aussi être méduse - une méduse au miroir, peut-être bien. 
Les pigeons blancs de la place Blanche laissaient des traces sur la neige, comme des plis sur un drap d'hôpital. Sur la peau blanche, un mal blanc toujours éclôt en un envers discret de la blancheur. Cela peut mettre très longtemps: il naît toujours, bouton de perceneige, et c'est un timbre ou une obole, un copyright ou une saxifrage.

Mes pigeons blancs s'étaient enfuis depuis vingt ans, depuis vingt-cinq. Reconvertis dans les juke-boxes ou enterrés dans les flippers, mes pigeons avaient rogné leurs ailes, mais les rémiges repoussent comme le trèfle à quatre feuilles, comme la saxifrage.

Je m'étais dit adieu, adolescent encore. Dans ma redoute à l'abandon, je retrouvais bien tard mon plexus de journal - de journal militant. Sérigraphies, tracts, pétitions. Dans des nuages d'un rose pâle et encore très peu vénérien, certes, dans une fièvre à peine marquée par le thermomètre à mercure, mais dans la fièvre, comme dans les bals où je faisais tapisserie.

Retrouver, retrouvé. Ces mots jumeaux tintaient partout, tintaient sans cesse, ils résonnaient dans ma douleur comme une aiguille d'acupuncteur, qui eût été tout aussi bien la guimbarde d'un vacher. Et dans la vibration de cette lame ou cette aiguille, je ne parvenais pas à décider (le désirais-je, seulement?) ce qui tintait vers l'advenu, ce qui tendait vers l'avenir.

J'ai retrouvé: retrouvé tard mon plexus de journal, les mots des autres qui s'infiltraient comme autant de nouvelles tronquées du monde des vivants, paroles encore frêles, à peine ébauchées, comme des velléités d'échange ou comme des flèches indiquant le pêne d'une serrure. 
À retrouver, à retrouver encore: l'avoine folle des rencontres, peut-être, les gestes maladroits, toujours inopportuns, la voix douce qui dit le pus du mal blanc quotidien, la proximité feinte. Était-ce ma fatalité?

Retrouvé, retrouver, douleur et saxifrage: des mots venus d'ailleurs, mais qui s'étaient emparé de moi, et dont sans doute j'étais épris déjà.

Mes pierres se délitaient l'une après l'autre. Mon moteur s'emballait, menait grand bruit: je commençais à transpirer dans ma cuisine, au carrelage déjà largement négligé. Me revenaient quelques nuits d'Idumée, des poches de Priape, une tardive adolescence rocheuse, spongieuse. 


\section{8}

Il fallut bien en convenir: j'avais désormais des ratés, ma grande époque frigorifiée était finie, j'allais user du commerce des mots, profiter du commerce des hommes. Et ce n'était pas même une compromission: en moi comme en dehors de moi, je faisais eau de toutes parts, et mes murs d'un blanc sale s'écroulaient pesamment, suscitant des joyaux de poussière.

Il faisait nuit quand je sortis de ma bastide: le vieux présent parcheminé allumait ses étoiles et m'invita à entrer à l'auberge. Les souvenirs d'antan s'y attablaient avec les démons affriolants de l'heure et leur troussaient les jupes. Et pourquoi non?

Ailleurs, et ce n'était jamais très loin, c'était même tout près, des estropiés mouraient en masse, sans aucune distinction, mais avec un parfait naturel, en prononçant parfois quelques mots exotiques, tels des Danton au petit pied. Mais les serveurs de chopes allaient bon train et la fête, m'assurait-on, était aussi pour moi: je n'avais qu'à m'enrôler, qu'à m'asseoir sur le banc des vivants, et la première consommation était offerte. 
Même tiède, l'écume de la bière est aussi blanche que la mousse à raser, le stylo qui me tient à la main est dur comme le manche d'un blaireau. J'ai débranché à temps mon thermostat, devenu inutile: je sais aujourd'hui magnifier la ferveur, notre vie unanime.

II ne fait froid que dans le cœur des pauvres. 